\title{
Image Classification using Neural Networks and Random Forest for a Citizen Science Project
}

\author{
Marilyn Menecucci Ibaez ${ }^{a 1}$, Adenilson Carvalho ${ }^{b}$ and Fernando M. Ramos ${ }^{a}$ \\ ${ }^{a}$ National Institute for Space Research, São José dos Campos, SP, Brazil \\ ${ }^{b}$ National Center for Monitoring and Warning Disaster, São José dos Campos, SP, \\ Brazil \\ Received on October 28,2016 / accepted on January 06, 2017
}

\begin{abstract}
Recently, a citizen science project called ForestWatchers [1] was launched in order to involve the citizens without experience in the monitoring of deforestation. Through a Web interface, volunteers from around the world are invited to review MODIS images of forest regions. Considering the large area worldwide covered by tropical forest, it is essential to use a fast classifier that meets a double objective: the pixel mapping into two classes ('Forest' and 'Non-forest') and the selection of pixels to be sent to volunteers for inspection. This project investigates the use the deforestation pattern classification in the Brazilian Amazon using MODIS images. Deforestation maps were generated from various sizes, from different areas of the state of Rondonia. The results were validated with the results of PRODES project, which annually evaluates deforestation in the Brazilian Amazon.

Keywords: Neural Network, Image Processing, Computing Citizen, Desforestation, Citizen Science, Random Forest.
\end{abstract}

\section{Introduction}

Since 1988, the program PRODES (Monitoring the Brazilian Amazon Forest Satellite) uses a semi-automated process and TM/Landsat images for estimating the annual deforestation rate in the Amazon. More recently, Deter program (Detection System Deforestation in Real Time) provides weekly alerts of deforestated areas with 25 hectares or more, using images from the MODIS (Moderate Resolution Imaging Spectroradiometer) satellite TERRA and ACQUA NASA. Recently, a citizen science project called ForestWatchers [1] was launched in order to involve lay citizens in deforestation monitoring tasks. Through a Web interface, volunteers from around the world are invited to inspect MODIS images and confirm if these areas have been correctly classified by the automatic classifier. Considering the large area covered by tropical forests, it is essential to develop a classifier that

\footnotetext{
${ }^{1}$ E-mail Corresponding Author: marilynmenecucciibanez@gmail.com
} 
meets a double objective: a fast pixel mapping into two classes ('Forest' and 'Non-forest') and the selection of pixels to be sent to volunteers for inspection, based on a trust metric. In this context, the aim of this work is to investigate the use of two different methods - neural network fuzzy MLPs (Multi-Layered Perceptrons) and Random Forest (RF) - for classification of deforestation patterns in the Brazilian Amazon using MODIS images. The goal of this research is to develop a fast classifier for mapping and selecting pixels to be sent to the volunteers of the project ForestWatchers. In order to test and compare the two approaches, we produced deforestation maps of various sizes of different areas of the state of Rondonia. The results are validated with the corresponding deforestation map of the PRODES project.

\section{Methods}

\subsection{Science Citizen}

Citizen cyberscience is a term used for a range of online projects in which individual volunteers or networks of volunteers, many of whom may have no specific scientific training, perform or manage research-related tasks such as observation, measurement or computation. It offers a low- cost way to both strengthen the scientific infrastructure and engage members of the public in science $[2]$.

ForestWatchers goal is to evaluate in a rigorous scientific fashion the benefits and limitations of public participation via the Web in deforestation monitoring tasks, by comparing results obtained from many volunteers, for example by statistical averaging or merit-based systems, with results from deforestation mapping by experts.

\subsection{Fuzzy Multilayer Perceptron}

The Multilayer Perceptron network has a simple interpretation as a form of input-output model, with the weights and biases as a model of free parameters. Such networks can model arbitrary complex functions with the number of layers and the number of units in each layer determining the complexity of the function. Learning of MLP network is performed using the back propagation algorithm. This algorithm is based on two basic steps: spreading an input pattern is displayed and its result is propagated layer by layer. At this stage the synaptic weights are fixed and the end is released a network output set, and retropagation at this stage the network output is compared at the output you want to have the error correction parameter. The weights are adjusted according to the result of error correction. This adjustment is applied layer by layer - the output layer to the input layer [3].

A Fuzzy Neural Network uses the parameters of a fuzzy system with the 
approximation techniques of a neural networks in information classification. The diffuse propagation of errors considers the relationship of the exact likeness generated by the diffuse desired output [6]. The use of MLPs with fuzzification level network and learning allows one to identify the pixels whose classification bears a higher degree of uncertainty.

In the project was used sigmoid activation function in MLP Neural Network and Fuzzy Neural nwtwork with three neurons in input layer, two hidden layer and one neuron in output layer. These properties were discovered using empirical tests to achieve the combination of properties that would generate the best result for the networks. Also tested different values for the momentum and learning rate data.

The Figure 1 show Computational Model of MLP Network.

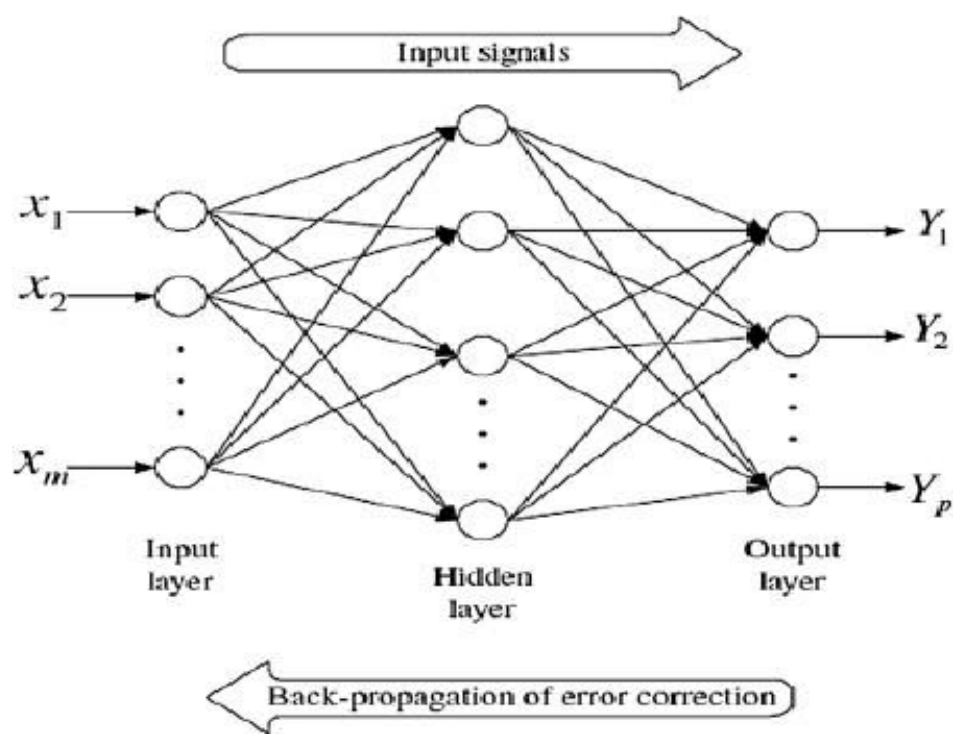

Figure 1: Computational Model of MLP Network.

Source: Adaptation [4].

where, $x_{1}, x_{2}, . ., x_{m}$ is input patterns and $y_{1}, y_{2}, \ldots, y_{m}$ is networkś output.

The Figure 2 show a Example of a diffuse multilayer Perceptron, na qual $x_{j, j=1, \ldots, M}-$ fuzzy variabel, $M-$ number of output, $A_{j}^{i}-$ fuzzy set, $T$ - actvation function, $g^{i}$ - real number defined in the output space, $N-$ number of rules. 


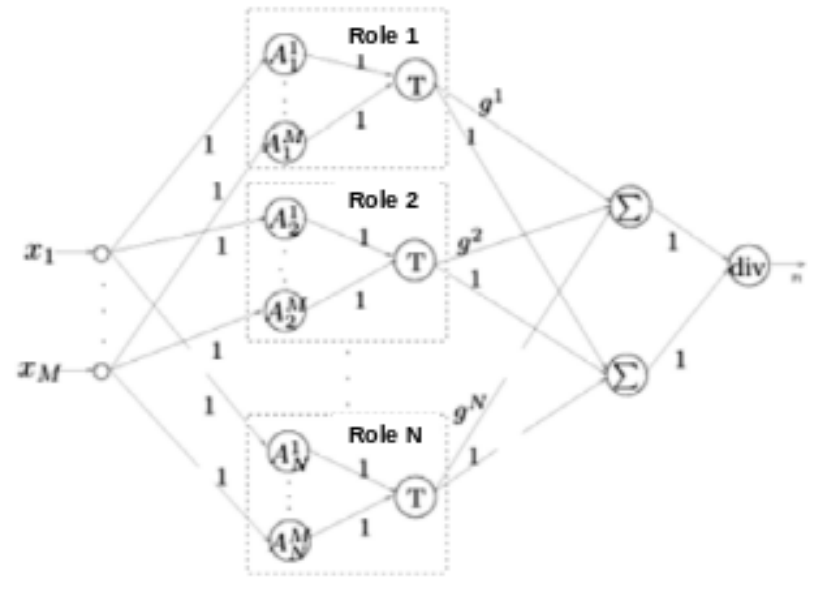

Source:[6].

Figure 2: Example of a diffuse multilayer Perceptron

\subsection{Random Forest}

Random Forest is a machine learning technique developed by [5]. This technique allows to obtain very effective models without any data preparation or knowledge modeling [5]. A Random Forest can be described as a classifier consisting of a set of decision trees $\left\{h\left(\mathbf{X}, v_{k}\right), k, 1, ..\right\}$, where $v_{k}$ are independently sampled random vectors, equally distributed in all the trees of the forest. The result of the classification process is the class $X$ with the highest number of votes among all the trees considered [5]. In this project was used Weka API Java and Random Forest in this API only permit to use classification tree.

\section{Methodology}

The methodology used for the classification of images in areas of forest and non-forest using the techniques can be summarized in Figure 3. 


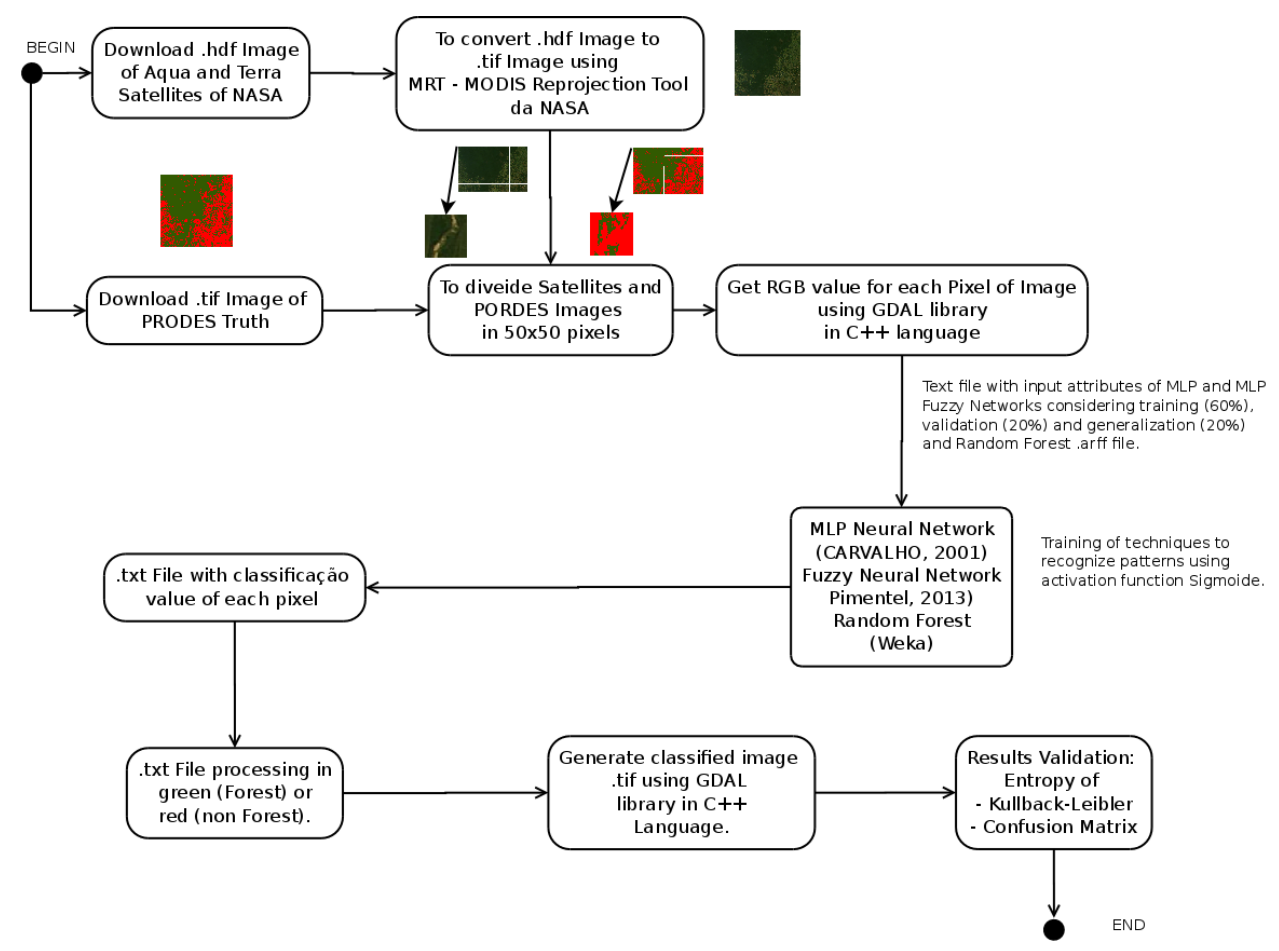

Figure 3: Methodology developed in project.

\section{Results}

Project results were obtained by applying the methodology above to test models with 500,000 pixels (medium scale) and with 8,341,210 pixels (large scale). Tests results are detailed in the following sections.

Table 1: Medium scale image classification.

\begin{tabular}{|c|c|c|c|c|}
\hline Id & PRODES Truth & MLP & Fuzzy MLP & $\begin{array}{c}\text { Random Forest } \\
500 \text { Trees }\end{array}$ \\
\hline & & & & \\
& & & & \\
1 & & & & \\
\hline
\end{tabular}


Table 2: Kullback-Leiber entropy for medium scale test.

\begin{tabular}{|c|c|c|c|}
\hline Id & MLP & Fuzzy MLP & $\begin{array}{c}\text { Random Forest } \\
500 \text { Trees }\end{array}$ \\
\hline 1 & 0.154 & 0.0100 & 0.283 \\
\hline
\end{tabular}

Table 3: Confusion matrix for medium scale tests.

\begin{tabular}{|c|c|c|c|c|c|c|}
\hline Id & \multicolumn{2}{|c|}{ MLP } & \multicolumn{2}{c|}{ Fuzzy MLP } & \multicolumn{2}{c|}{$\begin{array}{c}\text { Random Forest } \\
500 \text { Trees }\end{array}$} \\
\hline \multirow{3}{*}{1} & $\mathrm{a}=\mathrm{NF}$ & $\mathrm{b}=\mathrm{F}$ & $\mathrm{a}=\mathrm{NF}$ & $\mathrm{b}=\mathrm{F}$ & $\mathrm{a}=\mathrm{NF}$ & $\mathrm{b}=\mathrm{F}$ \\
\cline { 2 - 7 } & 2194 & 200 & 849 & 1275 & 1528 & 804 \\
\cline { 2 - 3 } & 100 & 6 & & 255 & 90 & 78 \\
\hline
\end{tabular}

Table 4: Large scale image classification.

\begin{tabular}{|l|l|}
\hline PRODES Truth & \\
\hline 500 Trees & \\
\hline
\end{tabular}

In order to validate our methodology, we compared classification images of Rondonia, for the year 2011, with the corresponding PRODES truth. Since the actual pixel size in the classification image is different from those of the PRODES images, it was necessary to determine the correction factor for transforming a given number of pixels, classified in a particular class ( $\mathrm{F}$ or $\mathrm{NF}$ ), into an area value (in hectares), which can be compared to the 
value provided by PRODES project. This conversion factor was obtained only once via calibration with PRODES data, and used in all the other simulations. Figure 4 compares the classification results generated by a RF with PRODES data for seven municipalities in Rondonia, for the year of 2012. Errors range between $3 \%$ to $9 \%$.

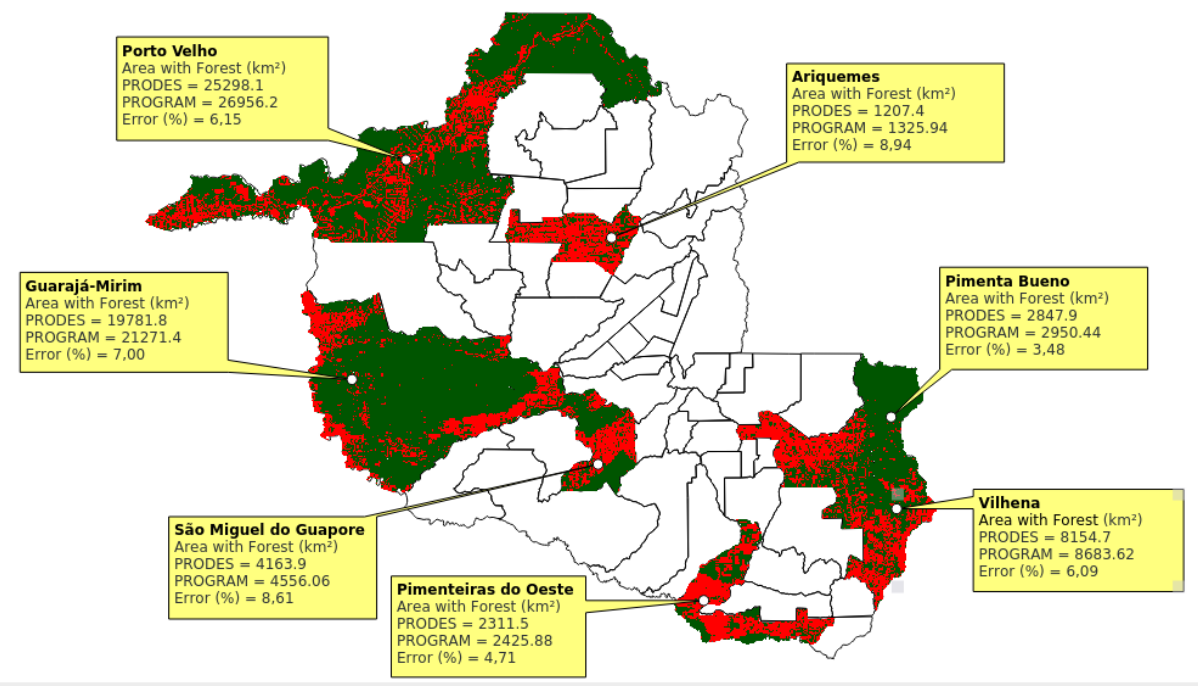

Figure 4: Comparison of forest area estimated by PRODES and the RF technique, for seven municipalities in RO, for the year of 2012.

\subsection{Calculation of uncertainty degree}

In order to decrease the number of pixels to be sent for analysis to volunteers, we computed the the degree of uncertainty $I$ of the classification of each pixel. Only pixels with a degree of uncertainty higher than a given threshold are sent for inspection. The degree of uncertainty is given by:

$$
I=\frac{|P-0.5|}{0.5},
$$

where $P$ is the ratio between the number of trees of the winning class and the total number of trees of the forest. Results are presented as gray-scale uncertainty y maps, since $I$ varies between 0 (white) and 1 (black).

We also calculate, for a given threshold, the redution coefficient of the number pixels that will be sent volunteers, where $C R=$ is coefficient of reduction, $P E V=$ number of pixels sent to volunteers and $P T I=$ total number of pixel of the classified image.

$$
C R=\left(1-\frac{P E V}{P T I}\right) * 100
$$


Table 5: Uncertainty map for the state of Rondonia in 2011 (500 trees)

\begin{tabular}{|c|c|c|c|c|}
\hline $\begin{array}{l}\text { Threshold } \\
\text { Uncertainty }\end{array}$ & $\begin{array}{c}\text { Map } \\
\text { Uncertainty }\end{array}$ & $\begin{array}{c}\text { Picture } \\
\text { Classified }\end{array}$ & $\begin{array}{l}\text { Posted } \\
\text { Image }\end{array}$ & $\begin{array}{l}\text { Reduction } \\
\text { Coefficient }\end{array}$ \\
\hline 0.20 & & & & $72.20 \%$ \\
\hline 0.40 & & & & $75.17 \%$ \\
\hline 0.70 & & & & $80.38 \%$ \\
\hline 0.90 & & & & $87.51 \%$ \\
\hline
\end{tabular}

\subsection{Estimation of Random Forest Processing Time}

Considering the large size of the forested areas, and, thus, the large size of the images to be analyzed and inspecyed, it is important to assess how the RF classifier processing time increases with the number of trees and the number of pixels. Results are presented in Figures 5 and 6 . It is observed that the computing time varies linearly (dashed black curve) with both the number of pixels and the number of trees, a good performance for classifying very large images. 


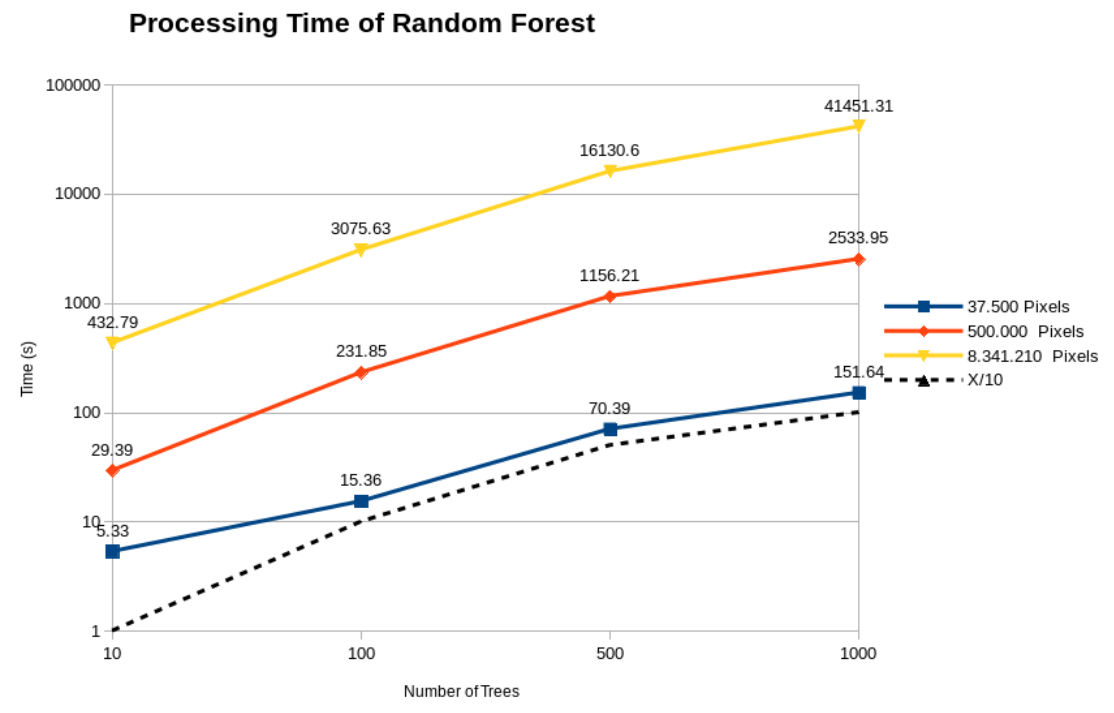

Figure 5: RF computation time as a function of the number of trees.

\section{Processing Time of Random Forest}

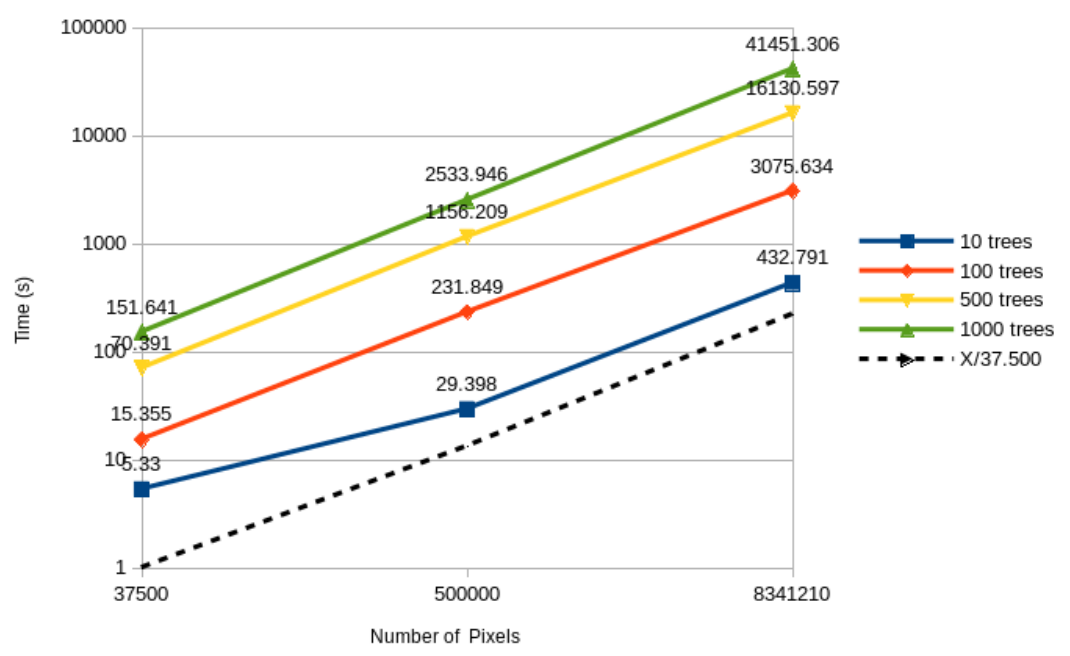

Figure 6: RF computation time as a function of the number of pixels. 
5. Conclusion In general, the RF classifier with 500 trees showed a better performance than the Fuzzy MLP and MLP. The RF classifier was evaluated in a large scale teste of estimating the area of forest in Rondonia in 2012. RF results have been compared to the PRODES truth and found to be in good agreement, with errors below $10 \%$. The RF also provided a simple metric of for the extraction of metric for the classification uncertainty, which permits a drastic reduction of the number of tasks to be sent for inspection to the volunteers. As for the computational time of the RF classification, it was observed that it grows only linearly with both the number of trees and the number of pixels of the image under analysis. These characteristics make the RF classifier an excellent choice to be used in the ForestWatchers citizen science project.

\section{References}

[1] LUZ, E. F. P.; CORRE, F. R.; GONZLEZ, D. L.; GREY, F.; RAMOS, F. M. The forestwatchers: a citizen cyberscience project for deforestation monitoring in the tropics. Humam Computation - A Transdiciplinary Journal, International Society for Optics and Photonics, v. 1, n. 2, p. 19, 2014.

[2] CONRAD, C. C.; HILCHEY, K. G. A review of citizen science and community-based environmental monitoring: issues and opportunities. Environmental Monitoring and Assessment, Springer, v.176, n. 4, p. 273291, 2011.

[3] HAYKIN, S. Redes neurais príncipios e práticas. 3. ed. Canad: McMaster University, 2001. 902 p. ISBN 978-85-7307-718-6.

[4] CARVAlho, A. R. Uso da redes neurais otimizadas para recuperação do perfil de concentração de gases traço atmosféricos a partir de dados de satélites. 208 p. Tese (Tese Doutorado em Computação) Instituto Nacional de Pesquisas Espaciais (INPE), Laboratório de Computação Aplicada - LAC Curso de Pós-Graduação em Computação Aplicada, So José dos Campos, 2011.

[5] BREIMAN, L. Random forests. Machine learning, Springer, v. 45, n. 1, p. $532,2001$.

[6] PIMENTEL, T. R. G. Master Degree Dissertation, INPE, 2016. sid.inpe.br/mtc-m21b/2014/02.28.18.48-TDI. 
